\title{
LABEL FIABILITE MESURES : UNE ALTERNATIVE POUR METTRE EN VALEUR LES COMPETENCES DES LABORATOIRES DE RECHERCHE
}

\author{
Lise Hégron \\ Centre Technologique Méditerranéen de Métrologie (CT2M) \\ Route de Lançon, Centre des Creusets 13250 SAINT-CHAMAS
}

\section{Résumé}

De nombreux laboratoires souhaitent mettre en place des dispositifs de maîtrise de la qualité des mesures et faire reconnaître leurs compétences. Toutefois, le niveau d'exigence de la norme NF EN ISO 17025 est peu adapté aux laboratoires et organismes de recherche, ainsi qu'aux laboratoires industriels de R\&D. En 2011, un groupe de travail constitué d'acteurs du milieu de la recherche publique et privée a développé un système d'exigences simplifié et adapté à leurs activités : le Label Fiabilité Mesures. Créé à l'origine pour permettre aux laboratoires de garantir la fiabilité des résultats et mettre en valeur leurs compétences, les deux laboratoires actuellement labellisés et les deux laboratoires en démarche avancée de labellisation s'accordent tous pour affirmer que le Label Fiabilité Mesures leur a apporté davantage.

\begin{abstract}
Many laboratories wish to improve the quality of measurements and get their skills recognized. However, the level of requirement of the testing and calibration standard ISO 17025 is poorly suited to research laboratories, as well as industrial R \& D laboratories. In 2011, a working group composed of players in the field of public and private research has developed a system that is simplified and adapted to the requirements of ISO 17025: " Label Fiabilté Mesures » (Measurements Reliability Label). Originally created to enable laboratories to ensure reliable results and enhance their expertise, both currently labellised laboratories and both soon labellised laboratories agree to say that the Label Fiabilité Mesures has brought them more.
\end{abstract}

\section{Introduction}

Un laboratoire souhaitant à ce jour garantir la fiabilité des résultats auprès de ses clients, assurer une traçabilité des informations et maintenir une pérennité de son activité se tourne majoritairement vers la NF EN ISO 17025 qui est un référentiel d'accréditation COFRAC pour les laboratoires d'étalonnages et d'essais. Néanmoins, le caractère inadapté de certaines exigences normatives pour le monde de la recherche, ainsi que le coût engendré par la mise en place et le maintien de l'accréditation provoquent l'hésitation de nombreux laboratoires à faire le pas vers ce référentiel. Un besoin subsiste pourtant en termes d'amélioration de la fiabilité des résultats ainsi que d'évaluation et de reconnaissance des compétences du laboratoire.

L'alternative proposée par le CT2M repose sur cette problématique et a abouti, après deux années de travail avec de nombreux acteurs du monde de la recherche, au Label Fiabilité Mesures. Ce dernier propose un référentiel basé sur deux niveaux.

Cet article s'attache principalement à développer le retour d'expérience des laboratoires actuellement labellisés et ceux en démarche de labellisation.

\section{$1 \quad$ Les origines de la démarche}

Les rencontres organisées entre 2009 et 2010 par le Centre Technologique Méditerranéen de Métrologie (CT2M) ont rassemblé des acteurs du monde de la recherche publique et privée (INRA, CIRAD, CEA, CNRS, INRETS, STMicroelectronics, CEMAGREF,...). Ces séances ont permis de mieux comprendre les besoins des laboratoires et les exigences qui leur semblaient les plus importantes. Les échanges ont ainsi abouti à l'élaboration du référentiel du Label 
Fiabilité Mesures contenant notamment des exigences jugées indispensables d'un point de vue technique [1], à savoir :

- la gestion des équipements de mesure,

- la maitrise des méthodes de mesure (validtion des méthodes et estimation des incertitudes de mesure),

- la traçabilité métrologique,

- la formation du personnel,

- la participation à des essais inter-laboratoires.

\section{Les principes du Label Fiabilité Mesures}

\subsection{Le référentiel}

Le référentiel du Label Fiabilité Mesures, inspiré de l'ISO 17025, regroupe des exigences relatives à la qualité ainsi que des exigences techniques. Le groupe de travail a mis en évidence des divergences entre les laboratoires au sujet de leurs systèmes qualité (plus ou moins en place), leurs objectifs et leurs investissements. C'est ainsi que le référentiel de labellisation a été créé sur la base d'un système à deux niveaux (Figure 1).

Le premier niveau, particulièrement adapté aux laboratoires de recherche entrant dans une démarche qualité, concerne les exigences de base considérées comme indispensables pour assurer la fiabilité des résultats de mesure.

Le second niveau de labellisation reprend les exigences du premier niveau auxquelles sont ajoutées (en bleu) des exigences supplémentaires permettant d'aller plus loin dans la maîtrise des processus de mesure ainsi que dans la mise en place d'un système de management de la qualité performant. Ce second niveau est adapté aux laboratoires désireux de faire reconnaître leurs compétences en termes de système qualité et de maîtrise technique.

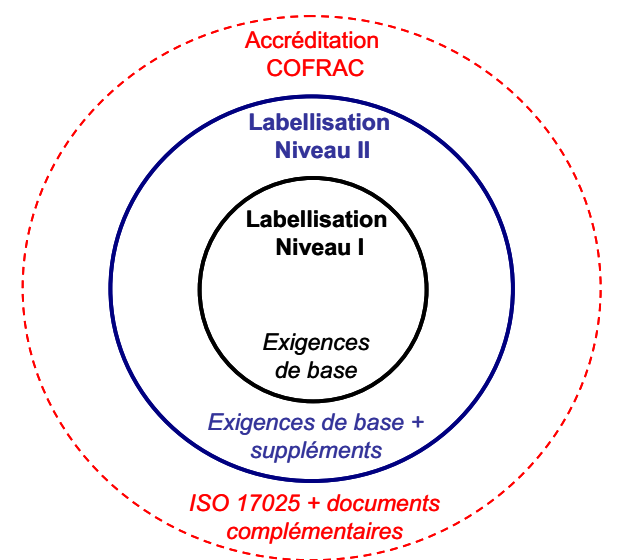

Figure 1 - Les deux niveaux de labellisation
Créé pour permettre aux laboratoires d'améliorer la fiabilité des résultats et faire reconnaître leurs compétences, le Label Fiabilité Mesures peut également être considéré comme un tremplin vers l'accréditation COFRAC. En effet, les exigences du référentiel de labellisation ont été définies de façon à s'intégrer parfaitement aux exigences de la norme ISO 17025.

\subsection{Le périmètre}

Le périmètre de labellisation doit être délimité de façon pertinente. L'entité labellisée peut être de différentes natures : un banc d'essai, un processus de mesure, une activité, une équipe de recherche, un laboratoire ou même un organisme. Chacun devra définir le périmètre en fonction de ses objectifs. Un laboratoire peut, par exemple, commencer par labelliser une analyse puis étendre le périmètre par la suite à l'ensemble de ses activités.

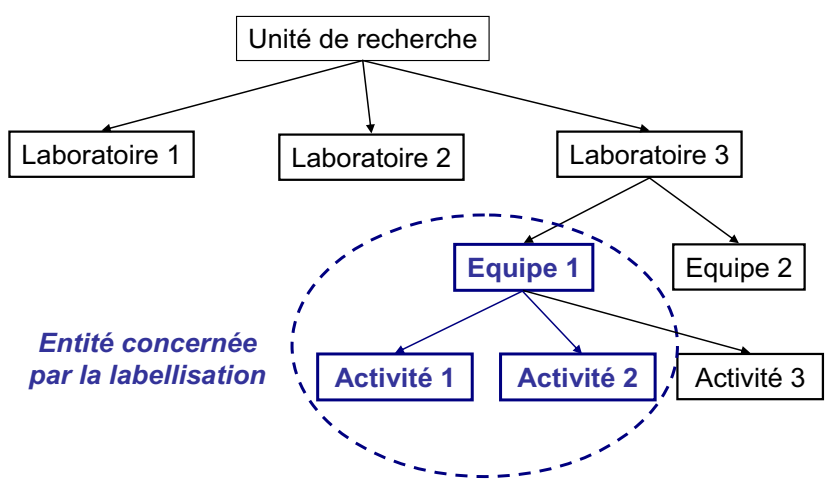

Figure 2 - Définition du périmètre de labellisation

\subsection{Organisation et fonctionnement du label}

L'organisation et le fonctionnement du label sont définis dans un règlement. Le label est délivré à l'issue d'un audit de labellisation et la décision est prise par un comité de labellisation. La labellisation est conservée sous réserve de transmettre le questionnaire d'auto-évaluation fourni par le CT2M ou le rapport d'audit interne annuel qui est une exigence de niveau I. Un audit de renouvellement de la labellisation est prévu tous les 3 ans. Le cycle d'audit est présenté sur la Figure 3. 


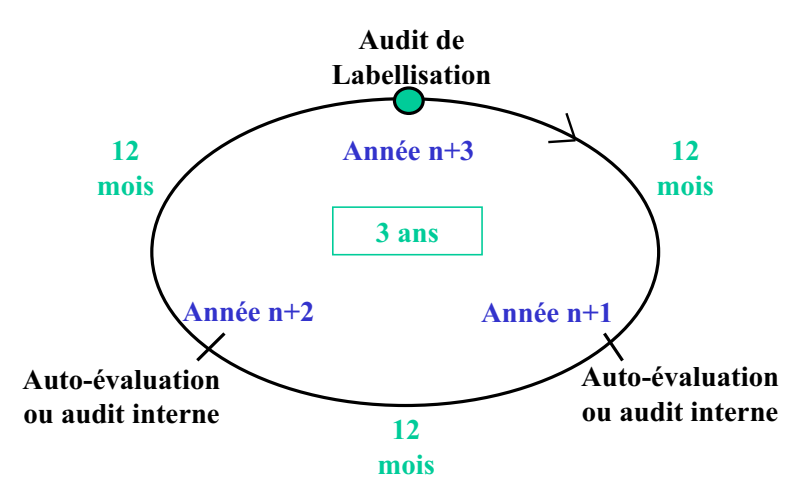

Figure 3 - Cycle d'audit de labellisation

L'ensemble des documents relatifs au LABEL FIABILITE MESURES sont disponibles sur le site internet du CT2M : www.ct2m.fr rubrique «Label Fiabilité Mesures » :

- Référentiel complet de labellisation,

- Formulaire de demande de labellisation,

- Constitution du comité de labellisation,

- Règlement du label,

- Formulaire de demande d'adhésion.

\section{Retour d'expérience des laboratoires}

Les laboratoires labellisés en 2015 sont au nombre de trois (Tableau 1), sachant qu'un laboratoire s'est vu destitué du Label Fiabilité Mesures en 2015 pour des raisons de mouvements de personnels clés. Deux autres laboratoires sont actuellement en démarche active de labellisation dont l'un d'entre eux devrait passer son audit initial courant 2015.

\begin{tabular}{|c|c|c|}
\hline NOMS & PERIMETRE & NIV. \\
\hline \multicolumn{3}{|c|}{ Entités labellisées } \\
\hline $\begin{array}{c}\text { CIRAD } \\
\text { Montpellier) }\end{array}$ & $\begin{array}{c}\text { Développement d'un kit de } \\
\text { laboratoire dans le cadre du } \\
\text { B. Sauret }\end{array}$ & I \\
\hline $\begin{array}{c}\text { IRSTEA } \\
\text { (Aix en Pce) }\end{array}$ & $\begin{array}{c}\text { Plateforme de Recherche } \\
\text { Technologique et } \\
\text { Géomécanique }\end{array}$ & I \\
\hline $\begin{array}{c}\text { INRA Byron } \\
\text { (Montpellier) }\end{array}$ & $\begin{array}{c}\text { Atelier des Isotopes Stables } \\
\text { M. P. Tillard }\end{array}$ & II \\
\hline Entités en démarche de labellisation \\
\hline $\begin{array}{c}\text { IM2NP } \\
\text { (Marseille) } \\
\text { Me } \text { C. Reynard- }\end{array}$ & $\begin{array}{c}\text { Instrumentation et Mesure pour } \\
\text { la quantification de } \\
\text { l'échauffement nucléaire en } \\
\text { réacteur de Type MTR }\end{array}$ & I \\
\hline $\begin{array}{c}\text { LMA } \\
\text { (Marseille) } \\
\text { M. C. Pinhède }\end{array}$ & $\begin{array}{c}\text { Essais en chambre anéchoïque } \\
\text { Earette }\end{array}$ & I \\
\hline
\end{tabular}

Tableau 1 - Laboratoires impliqués dans le Label Fiabilité Mesures

\subsection{Investissement initial}

L'entrée dans la démarche de labellisation demande un investissement qui dépend du niveau de labellisation demandé, du périmètre, du système qualité déjà en place, du nombre de personnes impliquées et du temps dégagé pour le personnel. Ainsi, entre un et trois ans sont nécessaires pour répondre de manière satisfaisante à toutes les exigences du référentiel (Figure 4). L'atteinte du niveau II est évidemment plus longue. Le laboratoire qui a demandé le Label Fiabilité mesures au niveau I et qui a eu besoin de plus de 2 ans pour répondre aux exigences n'a pas encore passé son audit initial de labellisation pour des raisons de déménagement.

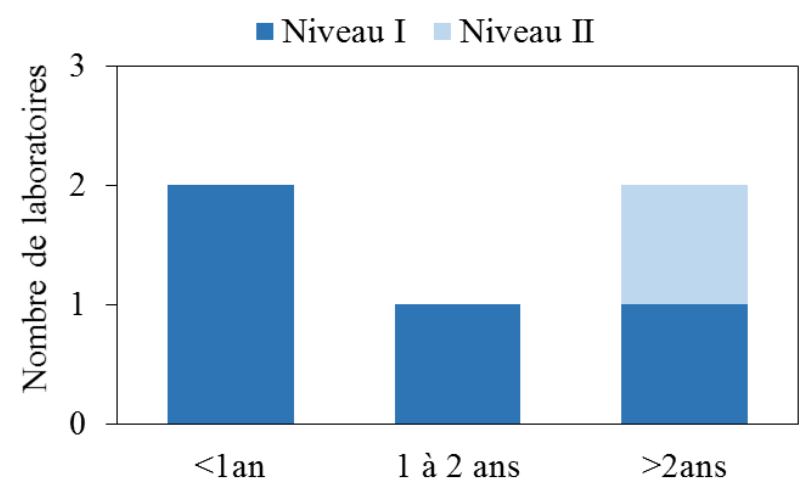

Figure 4 - Temps nécessaire entre le lancement de la démarche de labellisation et l'audit initial

Chaque laboratoire est évidemment accompagné par un consultant du CT2M et ce, à raison de deux à 3 jours entre la première réunion de diagnostic incluse et l'audit initial de labellisation (Figure 5).

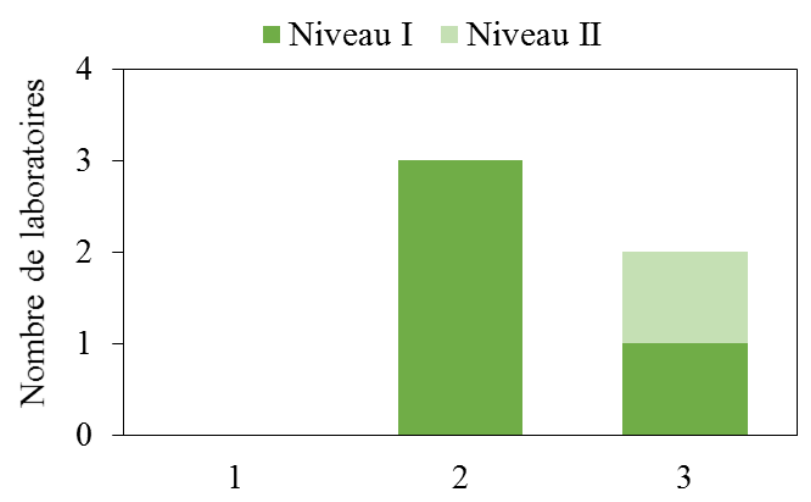

Figure 5 - Journées d'accompagnement/conseils par un consultant du CT2M

En majorité, les laboratoires estiment qu'ils ont eu besoin de plus de 20 journées pour répondre aux exigences du Label Fiabilité Mesures, quel que soit le niveau demandé (Figure 
6). Ce résultat est révélateur, d'une part de l'investissement et la motivation des personnes impliquées dans cette démarche et, d'autre part du manque de dispositions techniques et qualité initiales pour garantir des résultats fiables dans les laboratoires de recherche.

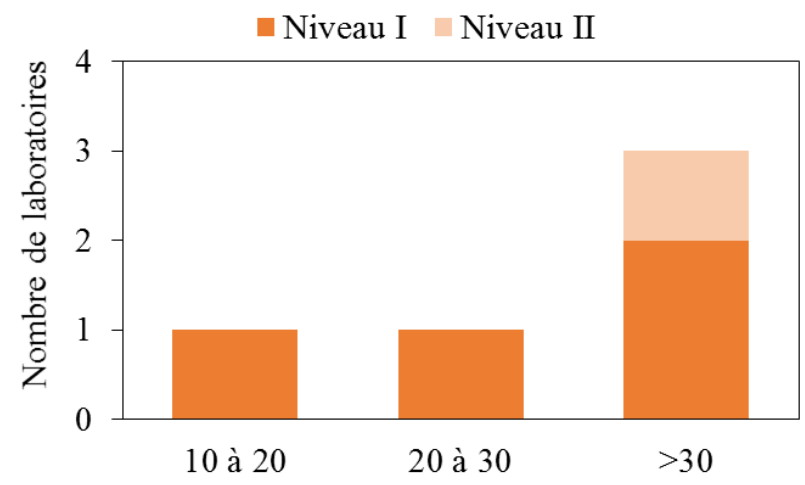

Figure 6 - Journées d'investissement pour répondre à l'ensemble des exigences du référentiel

Le coût financier tend vers une enveloppe globale inférieure à $5000 €$ pour la majorité des laboratoires (Figure 7). Un seul laboratoire a investi entre 5000 et $10000 €$.

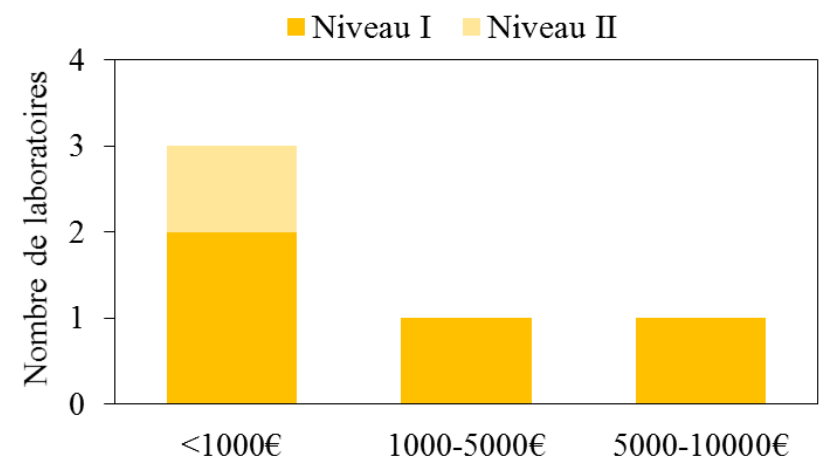

Figure 7 - Coût moyen pour répondre à l'ensemble des exigences du Label Fiabilité Mesures

\subsection{Les exigences du Référentiel}

Les laboratoires labellisés ou engagés dans la démarche ont répondu à un questionnaire qui a permis d'évaluer le travail fourni pour chacune des exigences du référentiel du Label Fiabilité Mesures. Les exigences (niveau I et niveau II) pour lesquelles ils ont dû mettre en place des dispositions, les formaliser dans des documents (manuel qualité, procédures, modes opératoires, ...) puis les appliquer sont :

- L'établissement d'une liste des documents en vigueur (\$2),
- L'identification de versions sur les documents (\$2),

- L'utilisation de fiches de non-conformités et d'actions correctives ( $\$ 3$ ),

- La formalisation de la demande du bénéficiaire des résultats (\$4),

- L'étalonnage des équipements critiques à une périodicité définie et la conservation des certificats d'étalonnage associés (\$10),

- Le suivi des équipements critiques au moyen de carte de contrôle (\$10),

- Les erreurs sur les enregistrements sont barrées et pas effacées (\$11),

- L'identification unique des échantillons (\$12).

Toutefois, les exigences qui n'ont nécessité que très peu de travail de la part des laboratoires sont, en majorité :

- La définition des responsabilités au sein du laboratoire à l'aide d'un organigramme $(\S 1)$,

- La revue de direction annuelle (\$6),

- Le dossier de chaque personnel contenant les informations telles que: $C V$, diplômes, attestation de formation, fiches d'entretien annuel (\$7),

- La fiche de poste pour chaque personnel décrivant les fonctions, responsabilités et habilitation ( $\$ 7$ ),

- Les besoins en formation du personnel (§7),

- La définition des spécifications des équipements et/ou consommables dans un document approprié, eg. un cahier des charge $(\S 8)$,

- La confidentialités et protection des données ( 99 ),

- La conservation des enregistrements ( 99$)$

- L'identification unique des équipements $(\S 10)$,

- La présence d'une fiche de vie et d'un dossier équipement à jour $(\$ 10)$.

Les exigences du niveau I semblent abordables pour la majorité des laboratoires et correspondent à des dispositions qui sont connues et appliquées mais manquent parfois de formalisation et/ou d'harmonisation entre les différentes personnes. 


\subsection{Apports de la démarche}

L'investissement pour obtenir le Label Fiabilité Mesures va au-delà de l'amélioration de la qualité des résultats et la reconnaissance de l'expertise de l'entité. En effet, même les entités qui ne sont pas encore labellisées relèvent des avantages qu'ils n'avaient pas prévus tels que l'implication du personnel, l'harmonisation des mesures, l'intérêt de formaliser de manière structurée les pratiques et la mise en place de nouvelles procédures. Globalement, ils mettent en évidence une meilleure gestion de leurs équipements et maîtrise des mesures, ainsi qu'une reconnaissance au sein de leur organisation et auprès de leurs collaborateurs.

Le Label Fiabilité Mesures est encore trop récent pour déterminer s'il attire de nouveaux clients/bénéficiaires des essais ou analyses. Il ne contribue néanmoins pas à les repousser !

\section{Conclusion}

Le Label Fiabilité Mesures satisfait à l'unanimité les entités qui le possèdent et celles qui sont engagées dans la démarche. Les laboratoires apprécient la cohérence des exigences du référentiel avec le monde de la recherche dans lequel ils travaillent. L'investissement initial concerne davantage du temps à dégager pour formaliser et mettre en application les exigences du référentiel qu'un budget à débloquer.

Vous pourrez ainsi trouver l'un des deux logos ci-dessous sur les documents et rapports d'essais des entités labellisées, le nombre d'étoiles correspondant au niveau du Label Fiabilité Mesures.

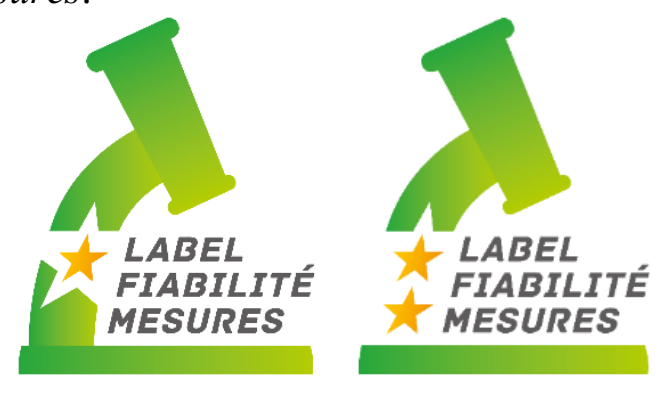

\section{Référence}

[1] Label fiabilités mesures : une alternative pour faire reconnaître les compétences des laboratoires de recherche, B. Geynet, Congrès International de Métrologie 2011. 